\title{
Information Sharing with Handheld Appliances
}

\author{
Jörg Roth \\ University of Hagen, Department for Computer Science \\ 58084 Hagen, Germany \\ Joerg.Roth@Fernuni-hagen.de
}

\begin{abstract}
Handheld appliances such as PDAs, organisers or electronic pens are currently very popular; they are used to enter and retrieve useful information, e.g., dates, to do lists, memos and addresses. They are viewed as stand-alone devices and are usually not connected to other handhelds, thus sharing data between two handhelds is very difficult. There exist rudimentary infrastructures to exchange data between handhelds, but they have not been designed for a seamless integration into handheld applications. Handheld devices are fundamentally different from desktop computers, a fact that leads to a number of issues. In this paper, we first analyse the specific characteristics of handheld devices, the corresponding applications and how users interact with handhelds. We identify three basic requirements: privacy, awareness and usability. Based on these considerations, we present our own approach.
\end{abstract}

\section{Introduction}

Currently, there exists a growing market for handheld devices such as PDAs, mobile phones, electronic pens etc. Upcoming communication technologies like UMTS and Bluetooth promise new functionalities for human communication. Currently, the mostly accepted way for communication is still verbal communication, whereas symbolic or textual media such as SMS or Email are still hard to use with handheld devices.

Handheld devices may already store reasonable amounts of data, e.g. appointments, holidays, addresses and agendas. On one hand, it should be possible to exchange these data between users without too much effort, on the other hand, handheld devices are basically of private nature and thus not intended for sharing data with other people. These contradictory characteristics may be one reason, why handhelds are currently being viewed as autonomous systems without any sophisticated facilities for inter-handheld communication.

In this paper, we present requirements, issues and problems of communicationoriented, distributed applications for handheld appliances. A lot of research has been done about the design of distributed applications in desktop environments. However, due to the fundamental different nature of handheld devices, these results can hardly be adapted to handheld scenarios.

This paper is structured as follows: first, we present the fundamental differences between handheld devices and traditional desktop computers. Based on these considerations we identify three key requirements, a communication-oriented distributed handheld application has to meet: privacy, awareness and usability. We then present a 
framework, which allows a designer to create successful information sharing applications for handhelds.

\section{Handheld Computing Characteristics}

The notion of handheld device, palmtop, PDA and organiser is often interpreted in different ways. An older interpretation distinguishes between pen-based devices and palmtops, where the latter have keyboards. In contrast, Microsoft divides Windows CE devices into handheld PCs (H/PC) with a keyboard, palmsize PCs (P/PC), which are controlled by a pen and handheld PC Pro devices, which are subnotebooks [3]. To get completely confused, Microsoft calls the new pen-based devices based on Windows CE 3.0 Pocket PC.

In the following, we understand by handheld devices mobile devices with small displays, without or with just rudimentary keyboards and an autonomous power supply. Examples are:

3Com's Palm devices (e.g. Palm III or Palm V),

- Casio's Cassiopeia or

_ Electronic pens such as the C-Pen.

In particular, we do not summarise notebooks or laptops under the notion of handhelds.

Handheld computing is closely related to so-called ubiquitous computing. Mark Weiser introduced the concept of ubiquitous computing often called "ubicomp" [19]. His vision was a huge number of invisible and "calm" computers surrounding people in their everyday life. In contrast, handheld computing keeps the device in the foreground, whereas ubicomp devices should work in the background [6]. People using handhelds are aware of using computers and adapt their activities to the device, e.g. learn a specific kind of handwriting. However, studying handheld computing may be the right step towards ubicomp, since a number of problems is identical. Handhelds, e.g., should be suitable for everyday tasks, easy to handle and fail proof.

Table 1 shows some hardware characteristics of popular handheld devices. Compared to desktop computers, handheld devices have small memories, low computational power, limited input and output facilities and usually do not contain a mass storage. Persistence data have to be stored in the battery-buffered RAM, and thus decreases the available runtime RAM for dynamic data and runtime stack. Even worse: some CPUs (e.g. Palms CPU) allow only addressing small pieces of memory $(64 \mathrm{k})$ as a whole. The reduced capabilities have two major reasons:

1. Size: The display is limited to palm size (approx. $10 \mathrm{~cm}$ x $8 \mathrm{~cm}$ ); chips (e.g. memory and CPU), even when highly integrated, need space for connectors and circuit boards. Due to these limitations, it is impossible or at least cost intensive to integrate high-resolution displays or a big number of electronic parts into a device.

2. Battery life: Fast CPUs, big memories and high-resolution displays (particularly coloured ones) consume a big amount of valuable battery power. If battery technology will not significantly improve in the future, handheld computers will always be far behind the capabilities of desktop computers. 
Table 1. Some hardware characteristics of handheld devices

\begin{tabular}{|l|l|l|l|l|}
\hline Device & Processor & RAM & Screen & Battery life \\
\hline $\begin{array}{l}\text { Palm IIIxe } \\
\text { (3Com) }\end{array}$ & $\begin{array}{l}16 \mathrm{Mhz} \\
\text { MC68EZ328 }\end{array}$ & $8 \mathrm{MB}$ & $\begin{array}{l}160 \times 160 \\
(16 \text { grey })\end{array}$ & $\begin{array}{l}1.5-2 \text { months } \\
\text { (normal use) }\end{array}$ \\
\hline $\begin{array}{l}\text { Cassiopeia E-15 } \\
\text { (Casio) }\end{array}$ & $\begin{array}{l}\text { 69MHz } \\
\text { NEC VR4111 }\end{array}$ & $16 \mathrm{MB}$ & $\begin{array}{l}240 \times 320 \\
(16 \text { grey) }\end{array}$ & $\begin{array}{l}25 \text { hours } \\
\text { (continuously) }\end{array}$ \\
\hline $\begin{array}{l}\text { C-Pen } 800 \\
\text { (C-Technologies) }\end{array}$ & $\begin{array}{l}100 \mathrm{Mhz} \\
\text { Intel StrongARM }\end{array}$ & $8 \mathrm{MB}$ & $\begin{array}{l}200 \times 56 \\
(\mathrm{~b} / \mathrm{w})\end{array}$ & $\begin{array}{l}2-3 \text { weeks } \\
\text { (normal use) }\end{array}$ \\
\hline
\end{tabular}

The reduced equipment of handheld devices has a big influence on software development. Handheld applications are usually not developed 'from scratch' but make use of their operating system's services. Popular operating systems like PalmOS [2], Windows CE [3], EPOC [17] or ARIPOS [5] provide the following services:

_ starting, stopping and switching applications, managing the memory and the user interface;

_ special device-dependent services like handwriting recognition, OCR, timedependent alarms;

_ supervising the battery power, performing auto-power-off;

- managing persistent data in the battery-buffered memory;

_ managing communication to other devices (usually to the host PC).

Software development kits allow the software designer to code and compile handheld applications on desktop computers, usually in C. Some kits provide emulators for testing applications before downloading them to the specific device.

Compared to desktop operating systems, handheld operating systems do not offer the same variety of services. Major shortcomings are:

Limited user interface capabilities: Usually the so-called WIMP paradigm (Windows, Icons, Menus, Pointers), which is very common to desktop computers, is, if at all, supported in a very reduced way only because of the limited display and input capabilities.

Limited support for persistent data: Since handhelds have no mass storage system, all persistent data have to be kept in the battery-buffer RAM. Windows CE emulates a hierarchical file system inside the RAM area. Other systems like ARIPOS and PalmOS store persistent data in so-called databases [2] (not to be confused with traditional databases). A database is a persistent collection of records. Each record has a unique identifier; its content is opaque to the operating system. Constructing and interpreting records solely depends on the corresponding application. Databases can be viewed as flat file system with a record-oriented structure.

Limited or no parallel execution capabilities: Most handheld operating systems do not support threads or processes for background tasks, a common technique for desktop computer applications. As a work-around, some systems offer so-called timers, which can periodically call a predefined procedure. Unfortunately, a call is only performed, when no other instruction is being executed, thus a timer does not provide real background operations.

Limited support for communication: Compared with desktop computers, handhelds only support a small set of communication capabilities. Handheld operating systems usually support one specific way of communication determined by the peripheral equipment. ARIPOS, e.g., only supports IrDA communication, since the C-Pen only 
contains an infrared transceiver for communication. PalmOS supports serial communication and TCP/IP, but does not support TCP server sockets, essential for reacting on incoming communication requests.

Networking: In addition to the limited communication support, the underlying network itself has some drawbacks:

- Wireless communication infrastructures currently have low bandwidths (GMS, e.g., only provides 9600 Baud) and tend to have high error rates and abnormal terminations.

_ Handhelds as communication end points are mobile in the network, i.e. often change their network addresses.

_ Mobile devices are rarely connected, i.e. are most of the time not available in the network because of network failures or just because the device is turned off.

Emerging technologies as UMTS, IPv6 and Bluetooth will change the way handhelds can be used inside a network. UMTS, e.g., allows a device to be permanently connected to the network with high bandwidth. IPv6 offers with MobileIP the possibility to keep the same IP address, even when a device moves inside the network. However, such technologies are not yet widely available and cannot be used inside current concepts.

All topics mentioned above have big influence on the application development process. Usually, because of the limited handheld capabilities developing handheld applications is very cost intensive. This includes testing and debugging; since handhelds do not offer the same debugging facilities like desktop computers, testing and debugging is quite cumbersome.

\section{Handheld Applications}

\subsection{Application Types}

Interactions with handheld applications and interactions with desktop applications follow different usage paradigms. First, handheld applications have to respect the limitations mentioned above; much more important: users request a different kind of availability from such applications: handhelds do not 'boot up'. When needed, applications have to immediately appear on screen. In turn, when the handheld device is deactivated, an application has to immediately save its state and disappear. In general, handheld applications are being used for entering and retrieving small pieces of information rather than processing data.

On handhelds, only a small set of application types can reasonably be used. We analysed 32 Palm applications currently available as shareware. In order to get a representative set of applications, we took a shareware collection of a popular German journal [4]. Table 2 shows the results.

Five applications are utilities and so-called hacks, which extend the operating system. This kind of application is not used to store information, thus not taken into further consideration. 
Table 2. A selection of Palm applications

\begin{tabular}{|l|l|l|l|}
\hline Applications & Application type & Data type & No. \\
\hline $\begin{array}{l}\text { Launcher III, SwitchHack, } \\
\text { Eerman Chars, Hackmaster, } \\
\text { Eco Hack }\end{array}$ & Utilities & mixed & 5 \\
\hline $\begin{array}{l}\text { Brainforest, dNote, } \\
\text { HandyShopper, } \\
\text { PocketMoney, HanDBase }\end{array}$ & $\begin{array}{l}\text { Textual notes, ideas, } \\
\text { shopping lists, bank } \\
\text { accounts }\end{array}$ & $\begin{array}{l}\text { textual } \\
\text { documents, } \\
\text { tables }\end{array}$ & 5 \\
\hline $\begin{array}{l}\text { Feiertage, Yearly, DateBk3, } \\
\text { Palm Planner }\end{array}$ & $\begin{array}{l}\text { Dates, appointments, } \\
\text { holidays }\end{array}$ & dates & 4 \\
\hline Abacus, TinySheet, MiniCalc & Spreadsheet tools & spreadsheets & 3 \\
\hline $\begin{array}{l}\text { Desktop to Go, Documents to } \\
\text { Go, TealDoc }\end{array}$ & Documents & $\begin{array}{l}\text { textual } \\
\text { documents }\end{array}$ & 3 \\
\hline ptelnet, MultiMail, HandWeb & Internet tools & mails, web pages & 3 \\
\hline DiddleBug, TealPaint & $\begin{array}{l}\text { Graphical notes, } \\
\text { freehand }\end{array}$ & graphical data & 2 \\
\hline Parens, Currency Calculator & Calculators & numbers & 2 \\
\hline PocketChess, TetrisV & Games & game states & 2 \\
\hline Secret! & Security & texts & 1 \\
\hline Route Europe & Route planner & geographic data & 1 \\
\hline Timer & Clock & time & 1 \\
\hline
\end{tabular}

Except for two programs, which allow graphical input, all applications store well structured and record oriented data, often text based. Typical data types for handheld applications are

texts and lists of texts,

- date entries,

- numbers,

_ tables or spreadsheets.

None of the applications above deals with multimedia data such as audio or video, which require a considerable network bandwidth, sufficient output devices and a huge amount of battery charge. Currently multimedia data are not suitable for handheld devices.

To summarise, most of the applications deal with simple data types such as strings or numbers, joined together to lists or tables. Only few applications support graphical data.

\subsection{Privacy}

Data stored inside a handheld device are usually viewed as private data. Even more than desktop computers, handhelds are viewed as personal devices [16] for storing personal data such as telephone numbers, birthdays and leisure-time activities. Some handheld operating systems protect private data. PalmOS, e.g., allows a user to mark entries as private, i.e. a password is needed for viewing them. In addition, a handheld device as a whole can be locked via a password. 
Connecting handheld devices for data exchange may jeopardize privacy. In case of an untrusted network, applications have to offer mechanisms for guaranteeing data. No private data should be transferred across a network, other people must not be able to break into a handheld and to spy out private data.

To gain acceptance by end-users, an infrastructure has not only to ensure privacy; but also convince the user that her or his private data are kept private. This is perhaps the most crucial issue related to privacy.

\subsection{Awareness}

Mobile devices can be connected to a network at different places. Depending on the location, different information may be available. Users should be aware of their current location, including the geographic location as well as the location in the network. This information is part of so-called context awareness. Abowd and Mynatt list different kinds of context awareness, defined by the "five W's": Who, What, Where, When and Why [1]. The Who context, e.g., is based on information about other people in the environment, especially for looking at activities.

Sharing information between people leads to the area of groupware and CSCW (computer supported collaborative work). Collaborative applications significantly differ from single-user applications. Many users provide input (often simultaneously), output has to be processed for many users, and shared data have to be kept consistent. Groupware applications have to provide a 'feeling' of working together in a group, called collaboration awareness: users have to be aware of other users involved in the collaborative task.

Context awareness as well as collaboration awareness require special components inside the applications' user interfaces called awareness widgets. Similar to users, we call an application aware of something, if it explicitly takes care of a special situation, otherwise we call it transparent. Collaboration aware applications, e.g., are especially designed for supporting a group, i.e. they contain special code for group functions. Collaboration transparent applications originally are single-user applications, which, with help of a groupware toolkit, can be used by many users simultaneously. Collaboration transparent applications do not offer awareness widgets. A similar notion can be applied to the mobility aspect: mobility aware applications contain code to handle mobility, e.g. react on unstable network connections and changing network locations. Mobility transparent applications cannot handle such problems explicitly, but rely on an underlying platform.

\subsection{Usability}

Usable applications support users in carrying out their tasks efficiently and effectively. For handheld applications, usability may be even more important than for desktop applications. When an application is designed in isolation from the intended users, the result is all too often an application which does not meet their needs and which is rejected by end-users. An application should meet the following requirements:

Respect hardware and software limitations: Usable applications take into account the hardware and software limitations of their hosting handheld devices. Heavy com- 
putational tasks are not suited for handhelds; user interfaces should be designed for small displays with minimal text input; communicating across a network should consider the small bandwidth and high error rate.

Software quality: Handheld applications should be more fail proof than desktop applications. A locked or crashed application blocks the entire device. An application trapped in an infinite loop prevents some devices from being switched off. Cold starting a handheld often results in loosing all stored data. The problem becomes even worse, if an application communicates with other devices. A blocked device may interrupt the entire group communication. To improve software quality, design guidelines may help a developer to build well-formed applications. Such a guideline can, e.g., be found in [2]. A platform or application framework, that encapsulates standard solutions for a specific application domain, helps a developer to meet these guidelines: She can rely on a set of services and only has to code application-specific functions.

Respect everyday requirements: Handheld applications are used every day. Abowd and Mynatt introduced the term called everyday computing [1]. They state that daily activities rarely have a clear beginning or end and often are being interrupted. This issue is especially important when considering communication-oriented applications. The strict distinction between asynchronous and synchronous groupware [7] hardly applies to everyday tasks. This leads to the notion of relaxed synchronous collaboration when group members collaborate synchronously, but may sometimes be disconnected from the network for short periods of time [15]. In addition, everyday tasks require spontaneous, unplanned communication. Exchanging data between handhelds should be as easy as a phone call. Especially, user-driven central co-ordination or administration should be avoided.

\section{Related Work}

Mobile phones: Mobile phones offer simple mechanisms to transfer textual data. SMS (short message service) [13] is a protocol, which allows sending up to 160 characters to another mobile phone. It can slightly be compared to the email service on the Internet, but is based on the mobile phone infrastructure GSM (global system for mobile communication). WAP (wireless application protocol) [20] allows browsing special Internet pages on small displays. WAP provides a one-way only information channel, i.e. it is not possible to send page contents from one device to another.

Beaming: A simple technique for exchanging data between handhelds, so-called "beaming", comes along with the Palm device [2]. Manufacturers of other devices, e.g. of C-Pens or Windows CE devices adapted the technology. Beaming can be viewed as de-facto standard for short range data exchange between handheld devices. It is infrared based and allows exchanging single records of data, e.g. one address or one memo. The distance between communicating devices should not exceed approx. one meter. Beaming requires human interaction, i.e. each time an entry is to be transferred, the sender as well as the receiver have to interact with their devices. Beaming is only suitable for small amounts of data.

PIMs: Personal Information Managers (PIMs) are important tools for handhelds. They conveniently allow entering data by keyboard and then downloading them to the handheld. A popular PIM is Microsoft's Outlook [11]. In addition to synchronising 
data with a handheld, Outlook allows scheduling appointments in a team, i.e. small amounts of data can be exchanged between a group of people.

Coda: Several research platforms have been developed for data distribution and consistency in mobile environments. Coda [9] provides a distributed file system similar to NFS, but in addition supports disconnected operations. Applications based on Coda are fully mobility transparent, i.e. run inside a mobile environment without any modification. Disconnected mobile nodes have access to remote files via a cache. Operations on files are logged and automatically applied to the server when the client reconnects. Coda applications can either define themselves mechanisms for detecting and resolving conflicts or ask the user in case of conflicts.

Rover: The Rover platform [8] supports mobility transparent as well as mobility aware applications. To run without modification, network-based applications such as Web browsers and news readers can use network proxies. The development of mobility aware applications is supported by two mechanisms: relocated dynamic objects (RDOs) and queued remote procedure calls (QRPC). RDOs contain mobile code and data and can reside on a server as well as on a mobile node. During disconnections, QRPCs are applied to cached RDOs. As in Coda, after reconnection operations are logged and applied to server data.

Bayou: Bayou [18] provides data distribution via a number of servers, thus segmented networks can be handled. In contrast to Coda, replicated records are still accessible, even when conflicts have been detected but not resolved. Bayou applications have to provide a conflict detection and resolution mechanism. Ideally, no user intervention is necessary. Bayou is not designed for supporting real-time applications.

Sync: Sync [12] supports asynchronous collaboration between mobile users. It provides collaboration based on shared objects, which can be derived from a Java library. As in Bayou, data conflicts are handled by the application. Sync applications have to provide a merge matrix, which for each pair of possible conflicting operations contains a resulting operation. With the help of the merge matrix, conflicts can be resolved automatically.

Lotus Notes: Lotus Notes [10] has not primarily been designed for mobile computers, but allows replicated data management in heterogeneous networks. Nodes can be disconnected and merge their data after reconnection. Data in Lotus Notes have a record structure. Fields may contain arbitrary data, which are transparent to Notes. Records can be read or changed on different nodes simultaneously. When reconnecting, users resolve conflicting updates. With the help of the Notes extension Mobile Notes, it is possible to access databases via Palm devices and mobile phones.

Discussion: Mobile phone protocols are designed for very simple data and not practical for structured data. It is difficult to adapt application specific data with an internal record structure to these protocols. A good solution for small amounts of data provides beaming, since any application can use this communication mechanism to exchange records with other handhelds. Due to the record-by-record concept, beaming is not suitable for bigger amounts of data.

Outlook has been designed especially for office environments and can hardly be adapted to other everyday tasks. It is not possible to add new applications to Outlook. In addition, Outlook requires a considerable amount of central administration.

Most of the research toolkits above request their mobile clients to be notebook computers with, e.g., hard disks. The focus of these platforms is to maintain data consistency in a weakly connected environment. Problems related to handheld devices such as small memory and reduced computational power are not handled satisfacto- 
rily. Automatic conflict detection and resolution need a considerable amount of resources on the handheld devices. We believe that such mechanisms are (currently) not suitable for handheld scenarios.

Concepts, such as the Rover toolkit, which require mobile code and marshalling/unmarshalling mechanisms, currently cannot be adapted to handheld devices, since they are significantly different from their servers. The concept of mobile code requires platform independent code and identical runtime libraries on both platforms involved. Even though languages such as Java are running on many platforms, handheld portings will provide other runtime libraries, thus mobile code mechanisms will fail.

The platforms above leave many problems described above unsolved. Especially privacy and awareness are still open issues.

\section{The QuickStep Approach}

The QuickStep platform [15] allows developing mobility aware and communicationoriented handheld applications. Developers can use communication and collaboration primitives provided by the platform and can concentrate on application-specific details. A set of predefined awareness widgets can be integrated into an application with a few lines of code. The QuickStep approach can be described as follows:

- QuickStep supports applications with well-structured, record oriented data. It explicitly has not been designed for supporting multimedia data, graphical oriented applications or continuous data streams.

_ QuickStep provides awareness widgets for collaboration awareness as well as context awareness.

_ QuickStep applications are fully collaboration and mobility aware.

_ QuickStep comes along with a generic server application, which allows supporting arbitrary client applications without modifying or reconfiguring the server.

_ The QuickStep architecture ensures privacy of individual data.

Before describing the QuickStep platform itself, we present two sample applications developed with QuickStep.

\subsection{Sample Applications}

The first sample application allows a group of users to exchange date information (e.g. about vacations or travellings). This tool is useful in meetings, in which members want to schedule appointments for future meetings. Each member owns a handheld device, which already contains a list of appointments as well as entries indicating the time one is unavailable. The problem is to find a date, when all members are available. Figure 1 presents an application that can help to find such a date.

The figure shows the view of two users on their personal handheld device. The upper half of the window displays the days of a month. Each range of dates when someone is unavailable is indicated by a bar. To get a better overview, the view can be switched to a two-months display. The lower half of the window serves as the legend for the upper half. 
a) Joerg's handheld

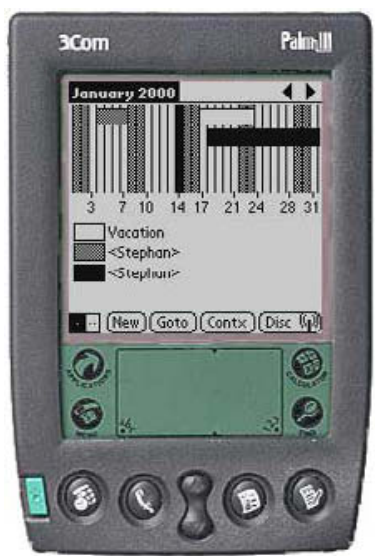

b) Stephan's handheld

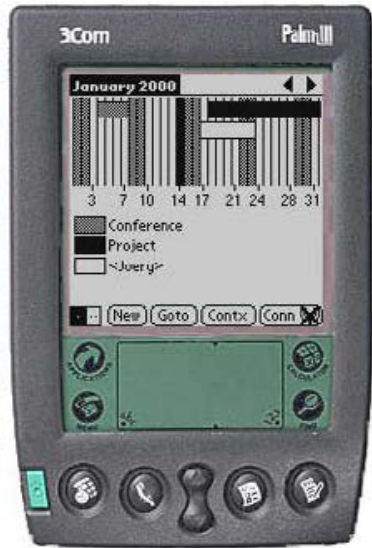

Fig. 1. A collaborative calendar tool

The two users Joerg and Stephan can see their own bars and the bars of each other. Foreign bars are labelled by the user name rather than the local label. For other users only the date range is of interest, not why someone is unavailable. Each user can make new entries which are distributed to the other user in real-time. With the help of this application, it is very easy to find dates, where all members are available.

The second example, the business card collector (figure 2), is a useful application for conferences. The application shows a list of all users assembled at a specific location. A user can view these cards and collect interesting cards in a persistent area. If the user permits, the business card collector publishes the card automatically, when entering a location.
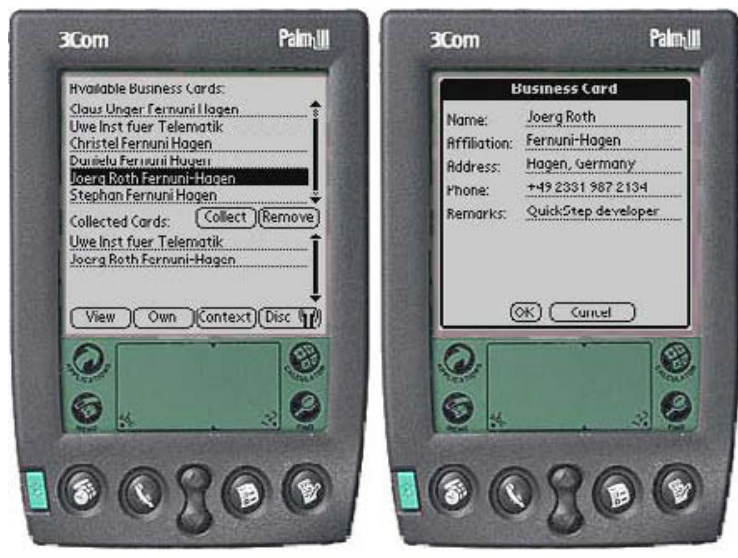

Fig. 2. A business card collector 
To develop such an application 'from-scratch', a developer has to implement many tasks, e.g., communication protocols have to be integrated, shared data have to be managed. The application should offer awareness widgets. All these services have to be developed in addition to the main task. Developing all these functions would overwhelm a developer. QuickStep helps a developer to concentrate on the application-specific details. Data primitives as well as predefined awareness widgets can be used from the platform.

In the following, we present the QuickStep platform. After describing the basic concepts, we discuss QuickStep with the help of the three key requirements privacy, awareness and usability.

\subsection{The QuickStep Infrastructure}

As described above, handheld operating systems offer only limited support for communication. Most systems cannot handle communication in the background. If the handheld device is always the initiating part of the communication, we need an additional computer, which acts as a communication relay between handhelds. This computer, the QuickStep server, contains a generic server application, which is able to serve arbitrary QuickStep applications.
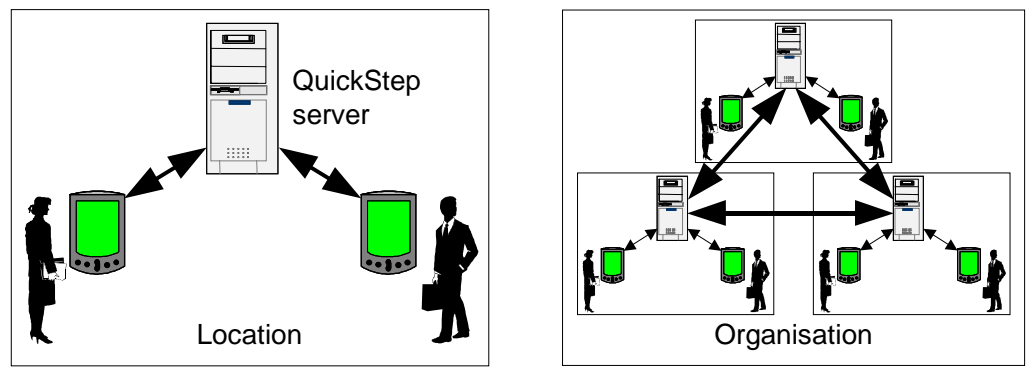

Fig. 3. QuickStep communication infrastructure

Figure 3 shows the QuickStep communication infrastructure. A QuickStep server operates in so-called locations. A location links all handheld devices together which are 'in range', i.e. which can be accessed by the specific communication technology. This can be the range of an infrared transceiver or a Bluetooth sender. Locations linked together form an organisation. Organisations may connect locations, which are in the same company, building, conference or public place. Table 3 shows typical examples for locations and organisations.

Table 3. Examples for locations and organisations

\begin{tabular}{|l|l|l|l|}
\hline & Train & Company & Conference \\
\hline Location & wagon & meeting room, hallway & presentation room, foyer \\
\hline Organisation & whole train & company building & whole conference \\
\hline
\end{tabular}


Connections between handhelds and QuickStep servers are usually wireless, where the QuickStep servers are connected via traditional local area networks. The QuickStep server can be viewed as 'inventory' of a specific location. Once installed, it normally has not to be reconfigured or administered. The server runs without an operator and does not need a user interface, thus can, e.g., work invisibly behind a panel.

\subsection{Underlying Data}

As mentioned above, most handheld operating systems offer an entity called database to handle application-specific data. The database is a common programming abstraction in handheld applications, thus the ideal abstraction for communication-oriented applications as well. QuickStep follows the same paradigm for collecting and distributing data. The QuickStep application programming interface (API) has similar database functions as the database API. An application developer can use well-known services to handle application specific data. Data stored in QuickStep databases are automatically distributed among a group by the QuickStep platform. Similar to native database services, the actual content of records is not of interest for the distribution mechanism and can only be interpreted by the application. Especially, the QuickStep server does not know the record structure.

Conflicts: Concurrent updates on shared data sometimes cause conflicts. Many platforms described above have complex mechanisms to detect and resolve conflicts. In our opinion, such mechanisms cannot be used inside handheld devices. Our concept for solving conflicts is simply to avoid them: it is not possible to concurrently manipulate data. For this, each record of data can only be changed by the handheld device, which originally created the record; copies residing on other handheld devices can only be viewed. To modify data which were created by another user, one has to make a private copy, which is treated as a new record.

Mirroring and Caching: Due to the low computational power of handhelds, heavy processing tasks should run on the server. On the other hand, with respect to the low network bandwidth, it is not possible to transfer a large amount of processing results between server and handheld. To reduce network traffic and to perform as many computations as possible on a server, we developed a combined mirroring and caching mechanism. Each handheld has its local database, which stores its user's data. A cache database stores all data of other users, the local user has currently in view. E.g., the cache database in the calendar tool stores dates of other users in a specific month. Finally, the QuickStep server has a copy of each local database, the mirror database. The mirror and cache databases are incrementally updated, each time a handheld device is connected to the server. The application developer has not to worry about the cache and mirror databases; they are completely set up and maintained by the QuickStep runtime system.

\subsection{Developing with QuickStep}

Figure 4 shows the environment, in which a QuickStep application is embedded.

Applications developed with QuickStep use the QuickStep API as well as the API offered by the corresponding operating system. QuickStep is built upon the database 
communication and user interface APIs. QuickStep does not use the operating system's communication API directly, instead it uses an intermediate layer, called the network kernel framework. This layer, developed by the DreamTeam platform [14] offers a generic interface for communication services such as starting and stopping connections, transferring data etc. With the help of the network kernel framework, it is possible to exchange the underlying communication API without changing the QuickStep platform. E.g., one can exchange a TCP/IP communication by a direct serial or infrared connection and only has to adapt the network kernel framework.

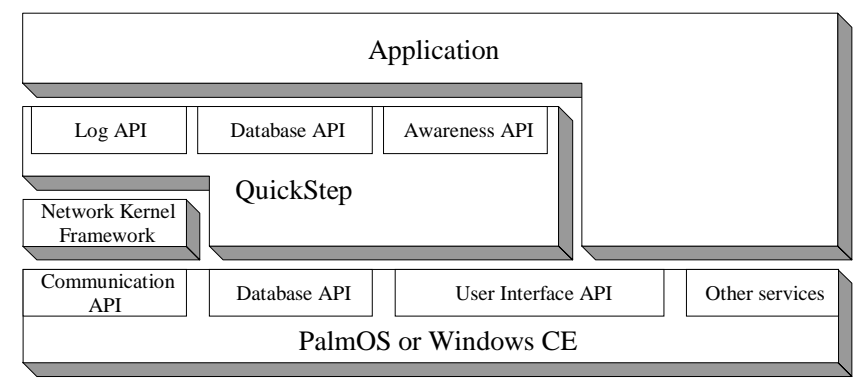

Fig. 4. The QuickStep programming environment

\subsection{Privacy}

To ensure privacy, QuickStep does not transfer any private data across the network. Every record can be marked as private (the default value). Private records reside only on the handheld and will not be transferred in any case.

Non-private records are not transferred until an anonymising process relieves them from personal information. Since the record structure is opaque to the underlying system, the anonymising function has to be provided by the application. In the calendar application, e.g., the anonymising function blanks out the labels of appointments and transfers the date range only. The entry

$$
\text { May 11-13: "Jörg is on the EHCI" }
$$

will be transformed to

$$
\text { May 11-13: "Jörg is away" }
$$

since others do not have to know anything about the reason of absence.

As an additional concept, each record has a 'time to live' entry, after which a record is deleted automatically from the QuickStep server and other handheld devices. This is done because a user wants to be sure that her or his data are not available forever on other computers (even in anonymised form). The time to life entry can be one of session, min, hour, day and forever. If the value is session, the corresponding record will be immediately removed from the server and handheld caches after the corresponding handheld is disconnected. The other values indicate the time, a record will reside after disconnection. The lifetime is controlled by special tasks inside the platform, the lifetime supervisors which exist on the handheld devices and on the QuickStep servers. 
A similar concept applies to the space property. Each record has a 'space to live' entry, which defines to which servers a corresponding copy is transferred. Space to live entries can be one of location, organisation and everywhere. If the value is location, only the QuickStep server which serves the current location gets a copy in the corresponding database. If the value is organisation, the record is transferred to all servers inside the organisation. The value everywhere is for future use and currently not supported. We work on a concept, which allows linking multiple organisations together for exchanging data. Currently, mirroring and group management requires a tight coupling between servers, thus is only suitable for local area networks. Transferring data between organisations requires completely different mechanisms.

\subsection{Awareness}

Context information is important for users as well as for the application, which may make decisions based on contextual data. A user who collaborates may want information about the context she or he is currently working in. For this, a user can open a frame as presented in figure 5.

The context frame is the central instance for all context-related information:

- What is the current connection state (connected or disconnected)?

- To which server is the handheld currently connected (server name, organisation)?

- What is my current location?

- Who can be called in case of problems (e.g. network failures)?

- Which other users are currently in the same location or organisation?

- What are other users' connection states?

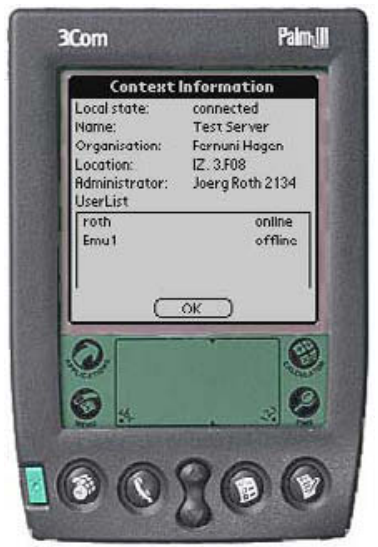

Fig. 5. The context frame

Location information is important when a user enters an unknown location. Consider a scenario where a huge building is equipped with a number of QuickStep servers (e.g. one per floor). Each QuickStep server provides information about the current location and thus can be used as a beacon for navigating inside the building. 
For collaborating users, the connection state is important. If a user is disconnected, data changes of that user cannot be viewed by other users. Thus, information about the connection state should be available on the main window of an application. We designed an integrated button and state indicator (figure 1, lower right button). This widget allows connecting and disconnecting to a QuickStep server and via a small icon indicates the current state.

The button/state indicator as well as the context frame are predefined awareness widgets and can be integrated in an application via the QuickStep library. In addition, an application can retrieve state and context information via the QuickStep API and can react on events (e.g. disconnecting from a network). So, an application developer can create his or her own awareness widgets.

\subsection{Usability}

QuickStep explicitly has been designed for handheld scenarios and respects hardware and software limitations. As described above, heavy computations are avoided and network limitations are considered. Using a well-formed and tested platform a developer can rely on stable services. The database abstraction offers a suitable application framework. All services related to communication are embedded into the platform. To easily find errors in the application itself, a developer can use the log API (see figure 4). Handheld logs are stored on QuickStep servers, thus problem analysis is possible, even when an application or the entire handheld device crashes.

Everyday tasks are often run in an unexpected way. In order to encourage spontaneous communication between users, central administration has to be avoided. For this, groups of interacting users are not defined explicitly in QuickStep. All users connected to a specific QuickStep server at the same time and using the same QuickStep application automatically form a collaborative session. This concept allows running a server without defining groups centrally. It is possible for a user to join a group without having explicit permission from other users. Since a mechanism for anonymising data is integrated into the platform, a user cannot spy out other users' private data. QuickStep does not provide services for leaving a collaborative group. When a user disconnects, the server first assumes a temporary disconnection, which happens frequently. Only if a user is disconnected for a longer time (e.g. an hour), the server removes that user from the session. The period of time, a user has to be disconnected until a leave operation is performed, is defined by the corresponding application. When a user leaves, the corresponding mirror database is deleted from the server.

\section{Conclusion and Future Work}

Handheld applications require approaches fundamentally different from desktop applications. If, in addition, applications have to exchange information between users, additional issues have to be considered. We identified three properties, an ideal communication-oriented handheld application has to meet: privacy, awareness and usability. 
The QuickStep approach meets these properties: it allows a designer to develop mobility and collaboration aware applications and has been especially designed for handheld devices. The generic QuickStep server relieves the handheld devices from heavy tasks and keeps data during disconnection. The QuickStep server operates without human intervention and can serve arbitrary QuickStep applications without modification. A server offers contextual information, which can be used by handheld applications. Data distribution is handled by a caching and mirroring mechanism.

In the future, we will follow two directions. First, we want to include traditional computers into the approach, because currently handheld computing relies on both, handheld and desktop applications. As data input is more convenient on desktop computers, an appropriate concept has to support both kinds of computers.

Second, we want to extend QuickStep to a global communication infrastructure. With this, two or more users operating at different places in the world could exchange data. Since wide area connections are considerably slow compared to local area networks, we have to develop new concepts. New technologies such as UMTS may help to address this problem.

\section{References}

1. Abowd G. D., Mynatt E. D.: Charting Past, Present and Future Research in Ubiquitous Computing, ACM Transactions on Computer-Human Interaction, Special Issue on HCI in the new Millennium, Vol. 7, No. 1, March 2000, 29-58

2. Bey C., Freeman E., Mulder D., Ostrem J.: Palm OS SDK Reference, 3Com, http://www. palm.com/devzone/index.html, Jan. 2000

3. Boling D.: Programming Windows CE, Microsoft Press, 1998

4. Brors D.: Software Highlights für Palm-Rechner, C'T Vol. 7, Apr. 2000, 138-141

5. C-Technologies, ARIPOS Programming, http://www.cpen.com

6. Demers A. J.: Research Issues in Ubiquitous Computing, Proc. of the thirteenth annual ACM symposium on Principles of distributed computing, Aug. 14-17, 1994, L.A., 2-8

7. Ellis C. A., Gibbs S. J., Rein G. L.: Groupware - some issues and experiences, Communications of the ACM, Vol. 34, No. 1, Jan. 1991, 39-58

8. Joseph A. D., Tauber J. A., Kaashoek M. F.: Mobile Computing with the Rover Toolkit, IEEE Transactions on Computers, Vol. 46, No. 3, March 1997 337-352

9. Kistler J. J., Satyanarayana M.: Disconnected Operation in the Coda File System, ACM Transaction on Computer Systems, Vol. 10, No. 1, Feb. 1992, 3-25

10. Lotus Development Corporation: Lotus Notes, http://www.lotus.com/home.nsf/welcome/ lotusnotes

11. Microsoft Outlook, http://www.microsoft.com/outlook

12. Munson J. P., Dewan P.: Sync: A Java Framework for Mobile Collaborative Applications, special issue on Executable Content in Java, IEEE Computer, 1997, 59-66

13. Point-to-point short message service support on mobile radio interface, http://www.etsi.org, Jan. 1993

14. Roth J.: DreamTeam - A Platform for Synchronous Collaborative Applications, AI \& Society (2000) Vol. 14, No. 1, Springer London, March 2000, 98-119

15. Roth J., Unger C.: Using handheld devices in synchronous collaborative scenarios, Second International Symposium on Handheld and Ubiquitous Computing 2000 (HUC2K), Bristol (UK), 25.-27. Sept. 2000

16. Stabell-Kulø T., Dillema F., Fallmyr T.: The Open-End Argument for Private Computing, First International Symposium on Handheld and Ubiquitous Computing, Karlsruhe, Germany, Sept. 1999, Springer, 124-136 
17. Tasker M., Dixon J., Shackman M., Richardson T., Forrest J.: Professional Symbian Programming: Mobile Solutions on the EPOC Platform, Wrox Press, 2000

18. Terry D. B., Theimer M. M., Petersen K., Demers A. J.: Managing Update Conflict in Bayou, a Weakly Connected Replicated Storage System, Proceedings of the fifteenth ACM symposium on Operating systems principles, Copper Mountain, CO USA, Dec. 3-6, $1995,172-182$

19. Weiser M.: The computer for the Twenty-First Century, Scientific American, 1996, Vol. 265, No. 3, Sept. 1991, 94-104

20. Wireless Application Protocol Architecture Specification, WAP Forum, http://www. wapforum.org/, April 30, 1998

\section{Discussion}

N. Graham: A lot of design decisions were based on limitations of the current technology. How many of those limitations are fundamental?

J. Roth: Some characteristics of handhelds will never change, e.g. display size. Display sizes will not improve since they have to fit in a hand. Another problem is battery life. This is only improving about $10 \%$ per year. So battery life will always be a limiting factor. There will certainly be faster CPUs in the future. Also wireless technology will also continue to be low bandwidth and higher error rates.

P. Van Roy: Your current design seems to rely on a server. 2 people who meet and would like to share information cannot if there is no server in the network environment. What if you went to a peer-peer style? How would you guarantee coherence? What would you do if there was no server; would people not be able to communicate? There exist protocols, e.g. Ginsella, that are serverless.

$J$. Roth: It is impossible to create a network that is always on because the devices are often switched off. So we need a server.

P. Van Roy: ... but gnutella doesnít require that devices are always on.

J. Roth: If two devices want to communicate then both devices need to be on.

S. Greenberg: Handheld devices are a real pain. I wish I had your stuff a few years ago. How do you handle how information propagated to other devices can be modified, that is, are people modifying the original data, or a copy of it? We ask this because in our own work with propagration of information over PDAs we noticed that people were sometimes confused over whether they were modifying someone elseís personal information or if they were modifying a public copy of this information. Unlike a piece of paper, you canít easily tell if it is a copy or the original. This is also a privacy issue. Have you come across something like this?

J. Roth: We avoid this problem. Persons have their own records. Only the originator of a piece of data is allowed to change it. Anyone can create or change their own information, but the ownership always remains with the originator and everyone can know who the owner of the information is.

F. Paterno: What kinds of building blocks/support do you have for developing applications, e.g. widgets, platform encapsulations?

J. Roth: We are only building an infrastructure, not a complete toolkit for creating applications. We donít offer UI widgets. 